\title{
Factor Group of a Fuzzy Soft Group
}

\author{
Anju S Mattam ${ }^{1}$, Dr. Sasi Gopalan ${ }^{2}$ \\ ${ }^{I}$ (Assistant Professor, Department of Mathematics, Little Flower College, Calicut University, Kerala, India) \\ ${ }^{2}$ (Associate Professor, Department of Mathematics, Cochin University of Science \& Technology, Kerala, India)
}

\begin{abstract}
In this paper we study Molodtsov's notion of Fuzzy Soft Sets considering the fact that the parameters(which are words or sentences) are mostly fuzzy hedges or fuzzy parameters. We discuss and study the properties and structural characteristics of fuzzy soft groups and normal fuzzy soft groups. Then we define the left coset, right coset and middle coset of a fuzzy soft group and prove some important results on them. Finally we present factor group of a fuzzy soft group.
\end{abstract}

Keywords: Fuzzy Soft Group, Fuzzy Soft Set, Normal Fuzzy soft group, Cosets of a Fuzzy soft Group, Factor Group of a Fuzzy soft Group

\section{Introduction}

Most of the existing mathematical tools for formal modeling, reasoning and computing are crisp, deterministic and precise in character. But in real life situation, the problems in economics, engineering, environment, social science, medical science, etc. do not always involve crisp data. Consequently, we cannot successfully use the traditional classical methods because of various types of uncertainities presented in these problems. There are several theories, for example theory of fuzzy sets[1], theory of intuitionistic fuzzy sets[2], theory of vague sets[3], theory of rough sets[4] which can be considered as mathematical tools for dealing with uncertainities. But all these theories have their inherent difficulties as pointed out by Molodtsov. Molodtsov initiated the concept of soft theory[5] as a new mathematical tool for dealing with uncertainities which is free from the above difficulties. Then P.K. Maji initiated the concept of fuzzy soft theory[6]. Rossenfeld proposed the concept of fuzzy groups[7] in order to establish the algebraic structures of fuzzy sets. This paper begins by introducing the basic concepts of fuzzy soft set theory. Then we introduce a basic version of fuzzy soft group theory which extends the notion of a group to the algebraic structures of fuzzy soft set.

Definition 2.1[8]

\section{Basic Definitions}

Let $X$ be the initial universe and $A$ be the set of parameters. Let $P(X)$ denote the power set of $X$. A pair (f,A) is called a Soft Set over X if $\mathrm{f}$ is a mapping from A into the set of all subsets of $\mathrm{X}$.

Definition 2.2[8]

Let $\mathrm{X}$ be a group and (f,A) be a soft set over $\mathrm{X}$ then (f,A) is called a soft group over $\mathrm{X}$, If and only if $\mathrm{f}(\mathrm{a})$ is a sub group of $\mathrm{X}, \forall a \in A$

Definition 2.3[9]

Let $\mathrm{I}^{\mathrm{X}}$ denote set of all fuzzy sets in $\mathrm{X}$. A pair (f,A) is called the fuzzy soft set if $\mathrm{f}$ is a mapping from $\mathrm{A}$ to the set of all fuzzy sets in X. i.e. $\mathrm{f}: \mathrm{A} \rightarrow \mathrm{I}^{\mathrm{X}}$

Definition 2.4[9]

A mapping T: I x I $\rightarrow$ I is called a t-norm defined on I x I, if the following conditions are satisfied

$\mathrm{T}(\mathrm{a}, 1)=\mathrm{a} \forall \mathrm{a} \in \mathrm{I}$

$\mathrm{T}(\mathrm{a}, \mathrm{b})=\mathrm{T}(\mathrm{b}, \mathrm{a}) \forall \mathrm{a}, \mathrm{b} \in \mathrm{I}$

$\mathrm{T}(\mathrm{a}, \mathrm{T}(\mathrm{b}, \mathrm{c}))=\mathrm{T}(\mathrm{T}(\mathrm{a}, \mathrm{b}), \mathrm{c}) \forall \mathrm{a}, \mathrm{b}, \mathrm{c} \in \mathrm{I}$

If $\mathrm{b} \leq \mathrm{c}$ then $\mathrm{T}(\mathrm{a}, \mathrm{b}) \leq \mathrm{T}(\mathrm{a}, \mathrm{c}) \forall \mathrm{a}, \mathrm{b}, \mathrm{c} \in \mathrm{I}$

If $\mathrm{T}(\mathrm{a}, \mathrm{a})=\mathrm{a}$, for arbitrary $\mathrm{a} \in \mathrm{I}$, then $\mathrm{T}$ is called an idempotent $\mathrm{t}$-norm

Definition 2.5[9]

Let $\mathrm{T}$ be an idempotent t-norm, $\mathrm{X}$ be a group and (f,A) be a fuzzy soft set over $\mathrm{X}$. Then (f,A) is said to be a fuzzy soft group over $\mathrm{X}$ iff for each $\mathrm{a} \in \mathrm{A}$ and $\forall \mathrm{x}, \mathrm{y} \in \mathrm{X}$.

$\mathrm{f}_{\mathrm{a}}(\mathrm{xy}) \geq \mathrm{T}\left(\mathrm{f}_{\mathrm{a}}(\mathrm{x}), \mathrm{f}_{\mathrm{a}}(\mathrm{y})\right)$ 
$\mathrm{f}_{\mathrm{a}}\left(\mathrm{x}^{-1}\right) \geq \mathrm{f}_{\mathrm{a}}(\mathrm{x})$

Example 2.6

Let $X=\{1, i,-1,-i\}$ and $A=\{a 1, a 2, a 3\} . X$ is a group under multiplication. Define $f: A \rightarrow I^{X}$ as

$(f, A)=\{f(a 1)=\{1 / 8, i / .4,-1 / .6,-i / .4\}$,

$$
\begin{aligned}
& \mathrm{f}(\mathrm{a} 2)=\{1 / .3, \mathrm{i} / .1,-1 /, 2,-\mathrm{i} / .1\}, \\
& \mathrm{f}(\mathrm{a} 3)=\{1 / .9, \mathrm{i} / .3,-1 / .6,-1 / .3\}\}
\end{aligned}
$$

We define $T\left(f_{a}(x), f_{a}(y)\right)=\min \left\{f_{a}(x), f_{a}(y)\right\}$, Then the pair (f,A) satisfies the conditions (5) and (6). Then (f,A) is a fuzzy soft group over $\mathrm{X}$.

Theorem 2.7[9]

Fuzzy soft group (f,A) over $\mathrm{X}$ satisfies

$\mathrm{f}_{\mathrm{a}}(\mathrm{e}) \geq \mathrm{f}_{\mathrm{a}}(\mathrm{x})$

$\mathrm{f}_{\mathrm{a}}\left(\mathrm{x}^{-1}\right)=\mathrm{f}_{\mathrm{a}}(\mathrm{x})$

Note 2.8

The maximum value $f_{a}(e)$ can take is 1 . So assigning this value to $f_{a}(e)$ and from condition (1) of definition2.4 we have $\quad \mathrm{T}\left(\mathrm{f}_{\mathrm{a}}(\mathrm{x}), \mathrm{f}_{\mathrm{a}}(\mathrm{e})\right)=\mathrm{f}_{\mathrm{a}}(\mathrm{x}), \forall \mathrm{x} \in \mathrm{X}$

Theorem 2.9[9]

Let $\mathrm{X}$ be a group and (f,A) be a fuzzy soft set over X. Then (f,A) is a fuzzy soft group iff for each

$\mathrm{a} \in \mathrm{A}$ and $\mathrm{x}, \mathrm{y} \in \mathrm{X}, \mathrm{f}_{\mathrm{a}}\left(\mathrm{xy}^{-1}\right) \geq \mathrm{T}\left(\mathrm{f}_{\mathrm{a}}(\mathrm{x}), \mathrm{f}_{\mathrm{a}}(\mathrm{y})\right)$

Definition 2.10

Let (f,A) be a fuzzy soft group over $\mathrm{X}$ and $\lambda \in(0,1]$ Then (f,A) is said to be a $\lambda$ - absolute fuzzy soft group over $\mathrm{X}$ if $\mathrm{f}_{\mathrm{a}}(\mathrm{x})=\lambda, \forall \mathrm{x} \in \mathrm{X}, \forall \mathrm{a} \in \mathrm{A}$

Definition 3.1

\section{Normal Fuzzy Soft Groups}

The fuzzy soft group (f,A) over $X$ is called a normal fuzzy soft group if for each $a \in A$,

$\mathrm{f}_{\mathrm{a}}\left(\mathrm{yxy}^{-1}\right)=\mathrm{f}_{\mathrm{a}}(\mathrm{x}), \forall \mathrm{x}, \mathrm{y} \in \mathrm{X}$

Definition 3.2

The fuzzy soft group (f,A) over $X$ is called an abelian fuzzy soft group over $X$ if for each a $\in A$,

$\mathrm{f}_{\mathrm{a}}(\mathrm{yx})=\mathrm{f}_{\mathrm{a}}(\mathrm{xy}), \forall \mathrm{x}, \mathrm{y} \in \mathrm{X}$

Definition 3.3

Let $\mathrm{X}$ be a group, (f,A) and (g,A) are two fuzzy soft groups over X. (f,A) is said to be conjugate to $(\mathrm{g}, \mathrm{A})$ if for each $\mathrm{a} \in A$ there exist $\mathrm{x}$ in $\mathrm{X}$ such that $\mathrm{f}_{\mathrm{a}}(\mathrm{y})=\mathrm{g}_{\mathrm{a}}\left(\mathrm{xy}^{-1}\right), \forall \mathrm{y} \in \mathrm{X}$.

Theorem 3.4

Fuzzy soft group (f,A) over $\mathrm{X}$ is normal iff it is conjugate to itself.

Proof: Proof is straight forward from the definitions 3.1 and 3.3

Theorem 3.5

Let (f,A) be a fuzzy soft group over $X$ and $y \in X$. Fuzzy soft set (g,A) over $X$ defined as for each a $\in$ $A, g_{a}(x)=f_{a}\left(y^{-1} x y\right)$ is a fuzzy soft group over $X$.

Proof: Let $r, s \in X$ and $a \in A$ 


$$
\begin{aligned}
g_{a}\left(r^{-1}\right) & =f_{a}\left(y^{-1} r s^{-1} y\right) \\
& =f_{a}\left(y^{-1} r y y^{-1} s^{-1} y\right) \\
& =f_{a}\left(\left(y^{-1} r y\right)\left(y^{-1} s^{-1} y\right)\right) \\
& \geq T\left(f_{a}\left(\left(y^{-1} r y\right), f_{a}\left(y^{-1} s^{-1} y\right)\right) \text { since(f, } A\right) \text { is a fuzzy soft group over } X \\
& =T\left(g_{a}(r), g_{a}(s)\right)
\end{aligned}
$$

hence by theorem $2.9(\mathrm{~g}, \mathrm{~A})$ is a fuzzy soft group over $\mathrm{X}$.

Theorem 3.6

If (f,A) be the normal fuzzy soft group over $X$, then for each $a \in A, H_{a}=\left\{h \in X: f_{a}(h)=f_{a}(e)\right\}$ is a normal subgroup of $\mathrm{X}$.

Proof: Let $\mathrm{x} \in \mathrm{X}$ and $\mathrm{h} \in \mathrm{H}_{\mathrm{a}}$. Since (f,A) is a normal fuzzy soft group over $\mathrm{X}$ we have

$\mathrm{f}_{\mathrm{a}}\left(\mathrm{xhx}^{-1}\right)=\mathrm{f}_{\mathrm{a}}(\mathrm{h}) \forall \mathrm{x}, \mathrm{h} \in \mathrm{X}$,

$\mathrm{f}_{\mathrm{a}}\left(\mathrm{xhx}^{-1}\right)=\mathrm{f}_{\mathrm{a}}(\mathrm{h})=\mathrm{f}_{\mathrm{a}}(e)$, since $\mathrm{h} \in \mathrm{H}_{\mathrm{a}}$

Therefore $\mathrm{xhx}^{-1} \in \mathrm{H}_{\mathrm{a}}$. Hence $\mathrm{H}_{\mathrm{a}}$ is a normal subgroup of $\mathrm{X}$.

Definition 3.7

Let (f,A) be a fuzzy soft group over $\mathrm{X}$. For each a $\in \mathrm{A}$, normalizer of the fuzzy soft group (f,A) is defined as $\mathrm{N}\left(\mathrm{f}_{\mathrm{a}}\right)=\left\{\mathrm{x} \in X: \mathrm{f}_{\mathrm{a}}\left(\mathrm{x}^{-1} \mathrm{yx}\right)=\mathrm{f}_{\mathrm{a}}(\mathrm{y}), \forall \mathrm{y} \in \mathrm{X}\right\}$

Theorem 3.8

For each a $\in \mathrm{A}$, normalizer of the fuzzy soft group (f,A) over $\mathrm{X}$ is a sub group of $\mathrm{X}$.

Proof: Let $a \in A$ and $r, s \in N\left(f_{a}\right)$ then we have

$$
\begin{aligned}
& \mathrm{f}_{\mathrm{a}}\left(\mathrm{r}^{-1} \mathrm{yr}\right)=\mathrm{f}_{\mathrm{a}}(\mathrm{y}) \text { and } \mathrm{f}_{\mathrm{a}}\left(\mathrm{s}^{-1} \mathrm{ys}\right)=\mathrm{f}_{\mathrm{a}}(\mathrm{y}) \\
& \begin{aligned}
\mathrm{f}_{\mathrm{a}}\left((\mathrm{rs})^{-1} \mathrm{yrs}\right) & =\mathrm{f}_{\mathrm{a}}\left(\mathrm{s}^{-1} \mathrm{r}^{-1} \mathrm{yrs}\right) \\
& =\mathrm{f}_{\mathrm{a}}\left(\mathrm{r}^{-1} \mathrm{yr}\right) \text { using }(12) \\
& =\mathrm{f}_{\mathrm{a}}(\mathrm{y})
\end{aligned}
\end{aligned}
$$

Therefore $r s \in \mathrm{N}\left(\mathrm{f}_{\mathrm{a}}\right)$

Let $r \in N\left(f_{a}\right)$

$$
\begin{aligned}
\mathrm{f}_{\mathrm{a}}\left(\left(\mathrm{r}^{-1}\right)^{-1} \mathrm{yr}^{-1}\right) & =\mathrm{f}_{\mathrm{a}}\left(\operatorname{ryr}^{-1}\right) \\
& =\mathrm{f}_{\mathrm{a}}\left(\mathrm{r}^{-1}\left(\operatorname{ryr}^{-1}\right) \mathrm{r}\right), \text { since } \mathrm{r} \in \mathrm{N}\left(\mathrm{f}_{\mathrm{a}}\right) \\
& =\mathrm{f}_{\mathrm{a}}(\mathrm{y})
\end{aligned}
$$

Therefore $\mathrm{r}^{-1} \in \mathrm{N}\left(\mathrm{f}_{\mathrm{a}}\right)$

Hence for each $\mathrm{a} \in \mathrm{A}$, normalizer of the fuzzy soft group (f,A) over $\mathrm{X}$ is a sub group of $\mathrm{X}$.

Theorem 3.9

$$
\text { Let (f,A) is a fuzzy soft group over } \mathrm{X} \text {. (f,A) is a normal fuzzy soft group over } \mathrm{X} \text { iff } \mathrm{N}\left(\mathrm{f}_{\mathrm{a}}\right)=\mathrm{X} \forall \text { a }
$$

$\in$ A.

Proof: Theorem follows from definition 3.1 and definition 3.7

Definition 4.1

\section{Cosets Of A Fuzzy Soft Group}

Let (f,A) be the fuzzy soft group over X.

Left coset of (f,A) determined by $\mathrm{X} \in \mathrm{X}$, (f,A) $\mathrm{L}_{\mathrm{L}(\mathrm{x})}$ is defined as for each

$\mathrm{a} \in \mathrm{A},\left(\mathrm{f}_{a}\right)_{\mathrm{L}(\mathrm{x})}(\mathrm{y})=\mathrm{f}_{a}\left(\mathrm{x}^{-1} \mathrm{y}\right), \forall \mathrm{y} \in \mathrm{X}$. 
Right coset of (f,A) determined by $\mathrm{x} \in \mathrm{X},(\mathrm{f}, \mathrm{A})_{\mathrm{R}(\mathrm{x})}$ is defined as for each

$\mathrm{a} \in \mathrm{A},\left(\mathrm{f}_{a}\right)_{\mathrm{R}(\mathrm{x})}(\mathrm{y})=\mathrm{f}_{a}\left(\mathrm{yx}^{-1}\right), \forall \mathrm{y} \in \mathrm{X}$.

Theorem 4.2

Let (f,A) be a fuzzy soft group over X. Then following statements are equivalent for each a $\in \mathrm{A}$ and $\forall \mathrm{x}, \mathrm{y} \in \mathrm{X}$

1) $f_{a}\left(x y x^{-1}\right)=f_{a}(y)$

2) $f_{a}(x y)=f_{a}(y x)$

3) $\left(f_{a}\right)_{L(x)}=\left(f_{a}\right)_{R(x)}$

Proof:

$(1) \Rightarrow(2)$

susbstituting y for $\mathrm{yx}$ in equation 1$)$ we get $\mathrm{f}_{\mathrm{a}}(\mathrm{xy})=\mathrm{f}_{\mathrm{a}}(\mathrm{yx})$

$(2) \Rightarrow(3)$

$\left(f_{a}\right)_{L(x)}(y)=f_{a}\left(x^{-1} y\right)=f_{a}\left(y x^{-1}\right)=\left(f_{a}\right)_{R(x)}(y)$

(3) $\Rightarrow(1)$

$f_{a}\left(x y x^{-1}\right)=\left(f_{a}\right)_{R(x)}(x y)=\left(f_{a}\right)_{L(x)}(x y)=\left(f_{a}\right)\left(x^{-1} x y\right)=f_{a}(y)$

Remark 4.3

From above theorem 4.2 we have (f,A) is a normal fuzzy soft group over $\mathrm{X}$ iff for each a $\in \mathrm{A}$,

$(\mathrm{f}, \mathrm{A})_{L(\mathrm{x})}=(\mathrm{f}, \mathrm{A})_{\mathrm{R}(\mathrm{x})}, \forall \mathrm{x} \in \mathrm{X}$

Example 4.4

Consider the fuzzy soft group in example 2.6. Using the definition 4.1

Right coset of (f,A) determined by 1 is given by $(\mathrm{f}, \mathrm{A})_{\mathrm{R}(1)}=(\mathrm{f}, \mathrm{A})$

Right coset of (f,A) determined by $\mathrm{i}$ is given

$$
\begin{aligned}
&(\mathrm{f}, \mathrm{A})_{R(i)}=\{ \mathrm{f}\left(\mathrm{a}_{1}\right)_{R(i)}=\{1 / .4, \mathrm{i} / .8,-1 / .4,-\mathrm{i} / .6\}, \\
& \mathrm{f}\left(\mathrm{a}_{2}\right)_{\mathrm{R}(\mathrm{i})}=\{1 / .1, \mathrm{i} / .3,-1 / .1,-\mathrm{i} / .2\}, \\
&\left.\mathrm{f}\left(\mathrm{a}_{3}\right)_{\mathrm{R}(\mathrm{i})}=\{1 / .3, \mathrm{i} / .9,-1 / .3,-\mathrm{i} / .6\}\right\}
\end{aligned}
$$

Right coset of (f,A) determined by -1 is given by

$$
\begin{aligned}
(f, A)_{R(-1)}=\{ & f\left(a_{1}\right)_{R(-1)}=\{1 / .6, i / .4,-1 / .8,-i / .4\}, \\
& f\left(a_{2}\right)_{R(-1)}=\{1 / .2, i / .1,-1 / .3,-1 / .1\}, \\
& \left.f\left(a_{3}\right)_{R(-1)}=\{1 / .6, i / .3,-1 / .9,-1 / .3\}\right\}
\end{aligned}
$$

Right coset of (f,A) determined by $-\mathrm{i}$ is given by

$$
\begin{aligned}
(f, A)_{R(-i)}=\{ & f\left(a_{1}\right)_{R(-i)}=\{1 / .4, i / .6,-1 / .4,-i / .8\}, \\
& f\left(a_{2}\right)_{R(-i)}=\{1 / .1, i / .2,-1 / .1,-i / .3\}, \\
& \left.f\left(a_{3}\right)_{R(-i)}=\{1 / .3, i / .6,-1 / .3,-i / .9\}\right\}
\end{aligned}
$$

Since(f,A) is a normal fuzzy soft group over $\mathrm{X}$, we have for each a $\in \mathrm{A},(\mathrm{f}, \mathrm{A})_{\mathrm{L}(\mathrm{x})}=(\mathrm{f}, \mathrm{A})_{\mathrm{R}(\mathrm{x})}, \forall \mathrm{x} \in \mathrm{X}$

Remark 4.5

If (f,A) is a $\lambda$ - absolute fuzzy soft group over $\mathrm{X}$, then all the cosets of (f,A) are $\lambda$ - absolute fuzzy soft groups over $X$. Otherwise coset of (f,A) determined by $x \in X$ is a fuzzy soft group only if $x=e$, the identity element of X.

Theorem 4.6

Let (f,A) be the fuzzy soft group over X such that $\mathrm{f}_{\mathrm{a}}(\mathrm{e})=1 \forall \mathrm{a} \in \mathrm{A}$. 
If for each $\mathrm{a} \in \mathrm{A}, \mathrm{H}_{1}=\left\{\mathrm{x} \in \mathrm{X}: \mathrm{f}_{\mathrm{a}}(\mathrm{x})=\mathrm{f}_{\mathrm{a}}(\mathrm{e})\right\}$ and $\mathrm{H}_{2}=\left\{\mathrm{x} \in \mathrm{X}:\left(\mathrm{f}_{\mathrm{a}}\right)_{\mathrm{R}(\mathrm{x})}(\mathrm{y})=\left(\mathrm{f}_{\mathrm{a}}\right)_{\mathrm{R}(\mathrm{e})}(\mathrm{y}), \forall \mathrm{y} \in \mathrm{X}\right\}$. Then $\mathrm{H}_{1}$ and $\mathrm{H}_{2}$ are sub groups of the group $\mathrm{X}$.

Proof: Let $\mathrm{x}, \mathrm{y} \in \mathrm{H}_{1}$

$$
\begin{aligned}
\mathrm{f}_{\mathrm{a}}\left(\mathrm{xy}^{-1}\right) & \geq \mathrm{T}\left(\mathrm{f}_{\mathrm{a}}(\mathrm{x}), \mathrm{f}_{\mathrm{a}}(y)\right) \quad \text { by theorem } 2.9 \\
& =\mathrm{T}\left(\mathrm{f}_{\mathrm{a}}(\mathrm{e}), \mathrm{f}_{\mathrm{a}}(\mathrm{e})\right) \quad \text { since } \mathrm{x}, \mathrm{y} \in \mathrm{H}_{1} \\
& =\mathrm{f}_{\mathrm{a}}(\mathrm{e})
\end{aligned}
$$

Since (f,A) is a fuzzy soft group we have using $(7) f_{a}(e) \geq f_{a}\left(x y^{-1}\right)$

From (13) and (14), we have $\mathrm{f}_{\mathrm{a}}(e)=\mathrm{f}_{\mathrm{a}}\left(\mathrm{xy}^{-1}\right)$

$\mathrm{xy}^{-1} \in \mathrm{H}_{1}$ and therefore $\mathrm{H}_{1}$ is a subgroup of $\mathrm{X}$.

Next we will prove that $\mathrm{H}_{1}=\mathrm{H}_{2}$

Let $\mathrm{h}_{1} \in \mathrm{H}_{1}$, Then $\mathrm{f}_{\mathrm{a}}\left(\mathrm{h}_{1}\right)=\mathrm{f}_{\mathrm{a}}(\mathrm{e})$

$$
\begin{aligned}
\forall \mathrm{y} \in \mathrm{X},\left(\mathrm{f}_{\mathrm{a}}\right)_{R\left(\mathrm{~h}_{\mathrm{1}}\right)}(\mathrm{y}) & =\mathrm{f}_{\mathrm{a}}\left(\mathrm{yh}_{1}^{-1}\right) \\
\geq & \mathrm{T}\left(\mathrm{f}_{\mathrm{a}}(\mathrm{y}), \mathrm{f}_{\mathrm{a}}\left(\mathrm{h}_{1}^{-1}\right)\right) \quad \operatorname{from}(5) \\
& =\mathrm{T}\left(\mathrm{f}_{\mathrm{a}}(\mathrm{y}), \mathrm{f}_{\mathrm{a}}(\mathrm{e})\right) \\
& =\mathrm{f}_{\mathrm{a}}(\mathrm{y}) \quad \text { from }(10) \\
& =\mathrm{f}_{\mathrm{a}}\left(\mathrm{ye}^{-1}\right) \\
& =\left(\mathrm{f}_{\mathrm{a}}\right)_{R(\mathrm{e})}(\mathrm{y}) \\
\left(\mathrm{f}_{\mathrm{a}}\right)_{R(\mathrm{e})}(\mathrm{y})=\mathrm{f}_{\mathrm{a}}\left(\mathrm{ye}^{-1}\right) & =\mathrm{f}_{\mathrm{a}}(\mathrm{y})=\mathrm{f}_{\mathrm{a}}\left(\mathrm{yh}_{1}^{-1} \mathrm{~h}_{1}\right) \\
& \geq \mathrm{T}\left(\mathrm{f}_{\mathrm{a}}\left(\mathrm{yh}_{1}^{-1}\right), \mathrm{f}_{\mathrm{a}}\left(\mathrm{h}_{1}\right)\right) \\
& =\mathrm{T}\left(\mathrm{f}_{\mathrm{a}}\left(\mathrm{yh}_{1}^{-1}\right), \mathrm{f}_{\mathrm{a}}(\mathrm{e})\right) \\
& =\mathrm{f}_{\mathrm{a}}\left(\mathrm{yh}_{1}^{-1}\right) \\
& =\left(\mathrm{f}_{\mathrm{a}}\right)_{R\left(\mathrm{~h}_{1}\right)}(\mathrm{y})
\end{aligned}
$$

from (15) and (16) we have $\left(\mathrm{f}_{\mathrm{a}}\right)_{R(\mathrm{e})}(\mathrm{y})=\left(\mathrm{f}_{\mathrm{a}}\right)_{R\left(\mathrm{~h}_{1}\right)}(\mathrm{y})$

$\therefore \mathrm{h}_{1} \in H_{2}$

$\Rightarrow \mathrm{H}_{1} \subseteq \mathrm{H}_{2}$

Let $\mathrm{h}_{2} \in \mathrm{H}_{2}$ then $\left(\mathrm{f}_{\mathrm{a}}\right)_{R\left(\mathrm{~h}_{2}\right)}(\mathrm{y})=\left(\mathrm{f}_{\mathrm{a}}\right)_{R(\mathrm{e})}(\mathrm{y}), \forall \mathrm{y} \in \mathrm{X}$

ie. $\mathrm{f}_{\mathrm{a}}\left(\mathrm{yh}_{2}^{-1}\right)=\mathrm{f}_{\mathrm{a}}(\mathrm{y}), \forall \mathrm{y} \in \mathrm{X}$

let $\mathrm{y}=\mathrm{e}$ then $\mathrm{f}_{\mathrm{a}}\left(\mathrm{h}_{2}{ }^{-1}\right)=\mathrm{f}_{\mathrm{a}}(\mathrm{e})$

$\Rightarrow \mathrm{h}_{2}^{-1} \in \mathrm{H}_{1}$

$\Rightarrow \mathrm{h}_{2} \in \mathrm{H}_{1}$, since $\mathrm{H}_{1}$ is a group

$\Rightarrow \mathrm{H}_{2} \subseteq \mathrm{H}_{1}$

Therefore $\mathrm{H}_{1}=\mathrm{H}_{2}$ (from(17) and (18))

since $H_{1}$ is a subgroup of $X$, we have $H_{2}$ also subgroup of $X$

Theorem 4.7

Let (f,A) be the fuzzy soft group over $\mathrm{X}$ such that $\mathrm{f}_{\mathrm{a}}(\mathrm{e})=1 \forall \mathrm{a} \in \mathrm{A}$.

If for each $\mathrm{a} \in \mathrm{A}, \mathrm{H}_{1}=\left\{\mathrm{x} \in \mathrm{X}: \mathrm{f}_{\mathrm{a}}(\mathrm{x})=\mathrm{f}_{\mathrm{a}}(\mathrm{e})\right\}$ and $\mathrm{H}_{2}=\left\{\mathrm{x} \in \mathrm{X}:\left(\mathrm{f}_{\mathrm{a}}\right)_{\mathrm{L}(\mathrm{x})}(\mathrm{y})=\left(\mathrm{f}_{\mathrm{a}}\right)_{\mathrm{L}(\mathrm{e})}(\mathrm{y}), \forall \mathrm{y} \in \mathrm{X}\right\}$.

Then $\mathrm{H}_{1}$ and $\mathrm{H}_{2}$ are sub groups of the group $\mathrm{X}$.

Proof: This theorem is proved similar to the proof of theorem 4.6 
Theorem 4.8

Let (f,A) be normal fuzzy soft group over $\mathrm{X}$ then for $\forall \mathrm{a} \in \mathrm{A}$

$\left(\mathrm{f}_{\mathrm{a}}\right)_{\mathrm{R}(\mathrm{x})}(\mathrm{xg})=\left(\mathrm{f}_{\mathrm{a}}\right)_{\mathrm{R}(\mathrm{x})}(\mathrm{gx})=\left(\mathrm{f}_{\mathrm{a}}\right)(\mathrm{g}), \forall \mathrm{g} \in \mathrm{X}$

Proof

$\left(\mathrm{f}_{\mathrm{a}}\right)_{\mathrm{R}(\mathrm{x})}(\mathrm{xg})=\left(\mathrm{f}_{\mathrm{a}}\right)\left(\mathrm{xgx}^{-1}\right)=\left(\mathrm{f}_{\mathrm{a}}\right)(\mathrm{g}), \forall \mathrm{g} \in \mathrm{X}$

$\left(\mathrm{f}_{\mathrm{a}}\right)_{\mathrm{R}(\mathrm{x})}(\mathrm{gx})=\left(\mathrm{f}_{\mathrm{a}}\right)\left(\mathrm{gXX}^{-1}\right)=\left(\mathrm{f}_{\mathrm{a}}\right)(\mathrm{ge})=\left(\mathrm{f}_{\mathrm{a}}\right)(\mathrm{g}), \forall \mathrm{g} \in \mathrm{X}$

Theorem 4.9

Let (f,A) be a fuzzy soft group over $\mathrm{X}$ such that $\mathrm{f}_{\mathrm{a}}(\mathrm{e})=1 \forall \mathrm{a} \in \mathrm{A}$. If for each a $\in \mathrm{A}$

$\left(\mathrm{f}_{\mathrm{a}}\right)_{\mathrm{R}(\mathrm{x})}=\left(\mathrm{f}_{\mathrm{a}}\right)_{\mathrm{R}(\mathrm{y})}$ then $\mathrm{f}_{\mathrm{a}}(\mathrm{x})=\mathrm{f}_{\mathrm{a}}(\mathrm{y}), \forall \mathrm{x}, \mathrm{y} \in \mathrm{X}$

Proof:

Given $\left(f_{a}\right)_{R(x)}=\left(f_{a}\right)_{R(y)}$

ie. $\mathrm{f}_{\mathrm{a}}\left(\mathrm{gx}^{-1}\right)=\mathrm{f}_{\mathrm{a}}\left(\mathrm{gy}^{-1}\right), \forall g \in X$

Substitute $\mathrm{g}=\mathrm{x}$ in (19)then $\mathrm{f}_{\mathrm{a}}(\mathrm{e})=\mathrm{f}_{\mathrm{a}}\left(\mathrm{xy}^{-1}\right)$

$$
\begin{aligned}
\mathrm{f}_{\mathrm{a}}(\mathrm{x}) & =\mathrm{f}_{\mathrm{a}}\left(\mathrm{xy}^{-1} \mathrm{y}\right) \\
& \geq \mathrm{T}\left(\mathrm{f}_{\mathrm{a}}\left(\mathrm{xy}^{-1}\right), \mathrm{f}_{\mathrm{a}}(\mathrm{y})\right) \\
& \geq \mathrm{T}\left(\mathrm{f}_{\mathrm{a}}(\mathrm{e}), \mathrm{f}_{\mathrm{a}}(\mathrm{y})\right) \\
& =\mathrm{f}_{\mathrm{a}}(\mathrm{y})
\end{aligned}
$$

Substitute $g=y$ in (19) then $\mathrm{f}_{\mathrm{a}}(\mathrm{e})=\mathrm{f}_{\mathrm{a}}\left(\mathrm{yx}^{-1}\right)$

$$
\begin{aligned}
& \mathrm{f}_{\mathrm{a}}(\mathrm{y})=\mathrm{f}_{\mathrm{a}}\left(\mathrm{yx}^{-1} \mathrm{x}\right) \\
& \geq \mathrm{T}\left(\mathrm{f}_{\mathrm{a}}\left(\mathrm{yx}^{-1}\right), \mathrm{f}_{\mathrm{a}}(\mathrm{x})\right) \\
& \geq \mathrm{T}\left(\mathrm{f}_{\mathrm{a}}(\mathrm{e}), \mathrm{f}_{\mathrm{a}}(\mathrm{x})\right) \\
&=\mathrm{f}_{\mathrm{a}}(\mathrm{x})
\end{aligned}
$$

From (20) and (21), $\mathrm{f}_{\mathrm{a}}(\mathrm{x})=\mathrm{f}_{\mathrm{a}}(\mathrm{y}), \forall \mathrm{x}, \mathrm{y} \in \mathrm{X}$

Definition 4.10

Let $(f, A)$ be the fuzzy soft group over X.

Middle coset of (f,A) determined by $\mathrm{x}, \mathrm{y} \in \mathrm{X},(\mathrm{f}, \mathrm{A})_{\mathrm{x}, \mathrm{y}}$ is defined as for each

$\mathrm{a} \in \mathrm{A},\left(\mathrm{f}_{a}\right)_{\mathrm{x}, \mathrm{y}}(\mathrm{z})=\mathrm{f}_{a}\left(\mathrm{x}^{-1} \mathrm{zy}^{-1}\right), \forall \mathrm{z} \in \mathrm{X}$.

Example 4.11

Middle coset of (f,A) determined by $1, i$ in example 2.6 is given

$$
\begin{aligned}
&(\mathrm{f}, \mathrm{A})_{1, \mathrm{i}}=\{ \mathrm{f}\left(\mathrm{a}_{1}\right)_{1, \mathrm{i}}=\{1 / .4, \mathrm{i} / .8,-1 / .4,-\mathrm{i} / .6\}, \\
& \mathrm{f}\left(\mathrm{a}_{2}\right)_{1, \mathrm{i}}=\{1 / .1, \mathrm{i} / .3,-1 / .1,-\mathrm{i} / .2\}, \\
&\left.\mathrm{f}\left(\mathrm{a}_{3}\right)_{1, \mathrm{i}}=\{1 / .3, \mathrm{i} / .9,-1 / .3,-\mathrm{i} / .6\}\right\}
\end{aligned}
$$

Theorem 4.12

Let (f,A) be the fuzzy soft group over $\mathrm{X}$ and $\mathrm{x}, \mathrm{y} \in \mathrm{X}$. If $\mathrm{x}=y^{-1}$ the middle coset of (f,A) determined by $\mathrm{x}, \mathrm{y}$ is a fuzzy soft group over X.

Proof 
For each $\mathrm{a} \in \mathrm{A}$ and $\mathrm{z}_{1}, \mathrm{z}_{2} \in \mathrm{X}$

$$
\begin{aligned}
& \left(\begin{array}{rl}
\left(f_{a}\right)_{x, y}\left(z_{1} z_{2}\right)= & \left(f_{a}\right)\left(x^{-1} z_{1} z_{2} y^{-1}\right) \\
= & \left(f_{a}\right)\left(x^{-1} z_{1} x x^{-1} z_{2} y^{-1}\right) \\
= & \left(f_{a}\right)\left(x^{-1} z_{1} y^{-1} x^{-1} z_{2} y^{-1}\right) \text {, since } x=y^{-1} \\
& \geq T\left(\left(f_{a}\right)\left(x^{-1} z_{1} y^{-1}\right),\left(f_{a}\right)\left(x^{-1} z_{2} y^{-1}\right)\right) \text {, since }(f, A) \text { is a fuzzy soft group } \\
& \geq T\left(\left(f_{a}\right)_{x, y}\left(z_{1}\right),\left(f_{a}\right)_{x, y}\left(z_{2}\right)\right) \\
\left(f_{a}\right)_{x, y} z^{-1}= & \left(f_{a}\right)\left(x^{-1} z^{-1} y^{-1}\right) \\
= & \left(f_{a}\right)(y z x)^{-1} \\
\geq & \left(f_{a}\right)(y z x) \\
= & f_{a}\left(x^{-1} z y^{-1}\right) \\
= & \left(f_{a}\right)_{x, y} z
\end{array}\right.
\end{aligned}
$$

$(\mathrm{f}, \mathrm{A})_{\mathrm{x}, \mathrm{y}}$ is a fuzzy soft group over $\mathrm{X}$.

Theorem 4.13 Factor Group of a Fuzzy soft Group

Let $\mathrm{X}$ be a group and (f,A) be the normal fuzzy soft group over $\mathrm{X}$ with $\mathrm{f}_{\mathrm{a}}(\mathrm{e})=1 \forall \mathrm{a} \in \mathrm{A}$. $\tilde{\mathrm{R}}$ be the set of all right cosets of (f,A) i.e. $\tilde{R}=\left\{(f, A)_{R(x)}: x \in G\right\}$ then $\tilde{R}$ is a group under the composition defined by $(\mathrm{f}, \mathrm{A})_{\mathrm{R}\left(\mathrm{x}_{1}\right)} \circ(\mathrm{f}, \mathrm{A})_{\mathrm{R}\left(\mathrm{x}_{2}\right)}=(\mathrm{f}, \mathrm{A})_{\mathrm{R}\left(\mathrm{x}_{1} \mathrm{x}_{2}\right)}$ i.e. for each $\mathrm{a} \in \mathrm{A}\left(\mathrm{f}_{\mathrm{a}}\right)_{\mathrm{R}\left(\mathrm{x}_{1}\right)} \circ\left(\mathrm{f}_{\mathrm{a}}\right)_{\mathrm{R}\left(\mathrm{x}_{2}\right)}=\left(\mathrm{f}_{\mathrm{a}}\right)_{\mathrm{R}\left(\mathrm{x}_{1} \mathrm{x}_{2}\right)} \quad \forall \mathrm{x}_{1}, \mathrm{x}_{2} \in \mathrm{X}$. R is called the factor group relative to the normal fuzzy soft group (f,A) over X.

Proof

We first prove that composition is well defined. Let $x, y, x_{0}, y_{0} \in G$ such that

$\left(\mathrm{f}_{\mathrm{a}}\right)_{\mathrm{R}(\mathrm{x})}=\left(\mathrm{f}_{\mathrm{a}}\right)_{\mathrm{R}\left(\mathrm{x}_{0}\right)} \&\left(\mathrm{f}_{\mathrm{a}}\right)_{\mathrm{R}(\mathrm{y})}=\left(\mathrm{f}_{\mathrm{a}}\right)_{\mathrm{R}\left(\mathrm{y}_{0}\right)}$

We have to show that $\left(\mathrm{f}_{\mathrm{a}}\right)_{\mathrm{R}(\mathrm{x})} \circ\left(\mathrm{f}_{\mathrm{a}}\right)_{\mathrm{R}(\mathrm{y})}=\left(\mathrm{f}_{\mathrm{a}}\right)_{\mathrm{R}\left(\mathrm{x}_{0}\right)} \circ\left(\mathrm{f}_{\mathrm{a}}\right)_{\mathrm{R}\left(\mathrm{y}_{0}\right)}$

i.e. $\left(f_{a}\right)_{R(x y)}=\left(f_{a}\right)_{R\left(x_{0} y_{0}\right)}$

i.e. $\left(\mathrm{f}_{\mathrm{a}}\right)\left(\mathrm{gy}^{-1} \mathrm{x}^{-1}\right)=\left(\mathrm{f}_{\mathrm{a}}\right)\left(\mathrm{gy}_{0}^{-1} \mathrm{x}_{0}^{-1}\right) \forall \mathrm{g} \in \mathrm{X}$

$$
\begin{aligned}
\left(f_{a}\right)\left(g y^{-1} x^{-1}\right) & =\left(f_{a}\right)\left(g y_{0}^{-1} y_{0} y^{-1} x^{-1}\right) \\
& =\left(f_{a}\right)\left(g y_{0}^{-1} x_{0}^{-1} x_{0} y_{0} y^{-1} x^{-1}\right) \\
& \geq T\left(f_{a}\left(g y_{0}^{-1} x_{0}^{-1}\right), f_{a}\left(x_{0} y_{0} y^{-1} x^{-1}\right)\right.
\end{aligned}
$$

From (22) we have $\mathrm{f}_{\mathrm{a}}\left(\mathrm{gx}^{-1}\right)=\mathrm{f}_{\mathrm{a}}\left(\mathrm{gx}_{0}^{-1}\right) \forall \mathrm{g} \in \mathrm{G}$

and $\mathrm{f}_{\mathrm{a}}\left(\mathrm{gy}^{-1}\right)=\mathrm{f}_{\mathrm{a}}\left(\mathrm{gy}_{0}^{-1}\right) \forall \mathrm{g} \in \mathrm{G}$

Substitute $g=\mathrm{x}_{0} \mathrm{y}_{0} \mathrm{y}^{-1}$ in (24) then

$$
\begin{aligned}
\mathrm{f}_{\mathrm{a}}\left(\mathrm{x}_{0} \mathrm{y}_{0} \mathrm{y}^{-1} \mathrm{x}^{-1}\right) & =\mathrm{f}_{\mathrm{a}}\left(\mathrm{x}_{0} \mathrm{y}_{0} \mathrm{y}^{-1} \mathrm{x}_{0}^{-1}\right) \\
& =\mathrm{f}_{\mathrm{a}}\left(\mathrm{y}_{0} \mathrm{y}^{-1}\right) \because(\mathrm{f}, \mathrm{A}) \text { is a normal fuzzy soft group } \\
& =\mathrm{f}_{\mathrm{a}}(\mathrm{e}) \text {, substituting } \mathrm{g}=\mathrm{y}_{0} \text { in }(25)
\end{aligned}
$$

From (23) we get $f_{a}\left(g y^{-1} x^{-1}\right) \geq T\left(f_{a}\left(g y_{0}^{-1} x_{0}^{-1}\right), f_{a}(e)\right)$

$$
=f_{a}\left(g_{0}^{-1} x_{0}^{-1}\right)
$$

$$
\mathrm{f}_{\mathrm{a}}\left(\mathrm{gy}_{0}^{-1} \mathrm{x}_{0}^{-1}\right)=\mathrm{f}_{\mathrm{a}}\left(\mathrm{gy}^{-1} \mathrm{yy}_{0}^{-1} \mathrm{x}_{0}^{-1}\right)
$$

$$
\begin{aligned}
& =f_{a}\left(g y^{-1} x^{-1} x_{y} y_{0}^{-1} x_{0}^{-1}\right) \\
& \geq T\left(f_{a}\left(g^{-1} x^{-1}\right), f_{a}\left(x y y_{0}^{-1} x_{0}^{-1}\right)\right.
\end{aligned}
$$


Substitute $\mathrm{g}=\mathrm{x} \mathrm{y} \mathrm{y}_{0}^{-1}$ in (24) then we get

$$
\begin{aligned}
\mathrm{f}_{\mathrm{a}}\left(\mathrm{xyy}_{0}^{-1} \mathrm{x}_{0}^{-1}\right)= & \mathrm{f}_{\mathrm{a}}\left(\mathrm{xyy}_{0}^{-1} \mathrm{x}^{-1}\right) \\
& =\mathrm{f}_{\mathrm{a}}\left(\mathrm{yy}_{0}^{-1}\right) \because(\mathrm{f}, \mathrm{A}) \text { is a normal fuzzy soft group } \\
& =\mathrm{f}_{\mathrm{a}}(\mathrm{e}) \text {, substituting } \mathrm{g}=\mathrm{y} \text { in }(25)
\end{aligned}
$$

From (27) we get $f_{a}\left(g y_{0}^{-1} x_{0}^{-1}\right) \geq T\left(f_{a}\left(g y^{-1} x^{-1}\right), f_{a}(e)\right)$

$$
=\mathrm{f}_{\mathrm{a}}\left(\mathrm{gy}^{-1} \mathrm{x}^{-1}\right)
$$

From (26) and (28) we get $\mathrm{f}_{\mathrm{a}}\left(\mathrm{gy}^{-1} \mathrm{x}^{-1}\right)=\mathrm{f}_{\mathrm{a}}\left(\mathrm{gy}_{0}^{-1} \mathrm{x}_{0}^{-1}\right)$

i.e. $\left(f_{a}\right)_{R(x y)}=\left(f_{a}\right)_{R\left(x_{0} y_{0}\right)}$ hence composition is well defined

Composition defined above is associative and $\left(\mathrm{f}_{\mathrm{a}}\right)_{\mathrm{R}(\mathrm{e})}$ is the identity element. Also we

have $\left(\mathrm{f}_{\mathrm{a}}\right)_{\mathrm{R}(\mathrm{x})} \circ\left(\mathrm{f}_{\mathrm{a}}\right)_{\mathrm{R}\left(\mathrm{x}^{-1}\right)}=\left(\mathrm{f}_{\mathrm{a}}\right)_{\mathrm{R}\left(\mathrm{x}^{-1}\right)} \circ\left(\mathrm{f}_{\mathrm{a}}\right)_{\mathrm{R}(\mathrm{x})}=\left(\mathrm{f}_{\mathrm{a}}\right)_{\mathrm{R}(\mathrm{e})}, \forall \mathrm{x} \in \mathrm{X}$. i.e inverse of $\left(\mathrm{f}_{\mathrm{a}}\right)_{\mathrm{R}(\mathrm{x})}$ is

$\left(f_{a}\right)_{R\left(x^{-1}\right)}$. Therefore $R$ is a group under the composition defined above.

\section{Conclusion}

We summarized the basic concepts of fuzzy soft group and then presented a detailed theoretical study of cosets of fuzzy soft group which led to the definition of factor group of a fuzzy soft group. This work focused on fuzzy soft groups, normal fuzzy soft groups and cosets of a fuzzy soft group. To extend this work one could study the properties of factor group of a fuzzy soft group.

\section{References}

[1]. H.J. Zimmerman, Fuzzy set theory and its applications, Kluwer Academic Publishers(Boston), 1996

[2]. K. Atanassov, "Intuitionistic fuzzy sets, " Fuzzy sets and systems, no.20, pp.87-96, 1986

[3]. W.L. Gan and D.J. Buehrer, "Vague Sets," IEEE Transactions Systems, Man and Cybernetics, Vol.23, no.2, pp.610- 614, 1993.

[4]. Z. Pawlak, "Rough Sets," International Journal of Information and computer science, no.11, pp.341-356,

[5]. 1982

[6]. D. Molodtsov, "Soft set theory-first results" Computer and Mathematics with application, no.37, pp.19-31,

[7]. 1999

[8]. P. K. Maji, R. Biswas and A.R. Roy, "Fuzzy Soft sets," The journal of fuzzy mathematics, Vol.3, no.9,

[9]. pp.589-602,2001

[10]. A. Rosenfeld, "Fuzzy Groups," Journal of Mathematical Analysis and Application, no.35, pp.512-517, 1971

[11]. Muhammad Aslam, Saquib Mazhes Qurashi, "Some contributions to soft groups" Annals of Fuzzy Mathematics and informatics, Vol. 4, no.1(july 2012) pp 177-195

[12]. Abdulkadir Ayyunoglu, Halis Aygun, "Introduction to Fuzzy Soft Groups" Computers and Mathematics with Application, no.58 (2009), pp.1279-1286 